\title{
Revisiting the Personality Traits and Dimensions within the Frame Work of Vedic Science
}

\author{
Radhey Shyam Kaushal ${ }^{1 *}$
}

\section{ABSTRACT}

The personality, as the outer garment of the character, manifests on different fronts of human actions and is assessed in an organization through several parameters, the so-called personality traits and dimensions. One popular approach used for this purpose is that of Jung which is based on four cognitive styles of psychology. The personality theory of Jung, in fact, yields four sharp categories of personality using two opposite ways of gathering information, namely sensation and intuition and another pair of two opposite ways of evaluating information, namely thinking and feeling. These different ways of gathering and evaluating information combine to form matrix of four problem-solving behaviors in cognitive psychology. Using the basics of Vedic Science, in particular the science of the Self (as developed earlier through the patomic model of human Being [1]), a deeper foundation to this scheme of classification of Jung is suggested in the present work. While investigating the ingredients of personality at the most fundamental level within this frame work, certain clues to improving the personality are also given. Further, with reference to an integrated personality (in terms of integrated body, mind and soul towards the success of an action) a personality creation mechanism is suggested which yields a new classification scheme on the basis of Vedic Science. It is argued that modern methods for assessing the personality while lack in assessing the spiritual content in the personality, the scheme suggested here, however, is rich enough for this purpose.

Keywords: Personality Traits, Dimensions, Frame Work, Vedic Science

Whether it is character building or the personality development, both mechanisms are mostly individual based in the sense that the external environment only offers a standard to judge their merit and not the method of their creation. While the former is somewhat subjective, like the inner garments of soul, the latter is objective like the outer garments. The character basically focussing on the distinctive human qualities, the personality, on the other hand, is an extreme

\footnotetext{
${ }^{1}$ Guest Faculty and Formerly UGC Research Scientist 'C', Department of Physics \& Astrophysics, University of Delhi, Delhi, India

*Responding Author

(C) 2016 I R Kaushal; licensee IJIP. This is an Open Access Research distributed under the terms of the Creative Commons Attribution License (http://creativecommons.org/licenses/by/2.0), which permits unrestricted use, distribution, and reproduction in any Medium, provided the original work is properly cited.
} 


\section{Revisiting the Personality Traits and Dimensions within the Frame Work of Vedic Science}

adulation of the individual. The character gives a boost to moral strength, the personality, however, invites both moral and physical strength in a varying proportion. However, both these aspects of human behavior originate from the same dynamics of inner essences of life (EOLs), termed here as the 'personality creation mechanism' (PCM).

In this paper, after presenting a brief survey of these aspects of human behavior studied in the modern context in the next section, the basic ingredients necessary for their creation are discussed in Sects.3 and 4. In Sect.5, we explore the possibilities of analyzing different personalities within the framework of Vedic science formulated earlier [1-3] by the author in terms of a philosophical atom-like (patomic) model of human Being and suggest some tips towards their improvement in Sect.6. Further, in Sect.7 we demonstrate a correspondence between two related aspects of human behaviour, namely 'personality creation' mechanism and the 'art of integrating' the body, mind and soul. An analysis of personalities within this framework is carried out in Sect. 8. Finally, concluding remarks are made in Sect.9.

While some mathematical preliminaries with regard to the dressing of EOLs with consciousness are given in Appendix A, we list here some abbreviations, notations and a glossary of some words used in the text. EFLs : essences for life; EOLs : essences of life appearing as per proposed [1] patomic (philosophical atom-like ) model of human Being consisting of biological body (B), senses of knowledge/perception and motor action (SE), mind (the faculty of feeling and emotions, M), intellect (the faculty of decision, I), ego (the faculty of memory or of selfsense, E), soul or spirit (the innermost existence, SO), and the external stimuli or worldly objects (WO) with which a human Being (b) interacts. Further, as per Vedic science [4] a human Being appears in three existences, namely causal or astral $(\mathcal{C})$, micro $(\mathcal{M})$, and gross $(\mathcal{G})$.

\section{STUDIES ON PERSONALITY AND ITS DEVELOPMENT IN MODERN TIMES}

Personality development has been a subject of study and research in psychology departments for more than a century now. In view of the downfall of moral and ethical values in the society as also an increase in the psychic diseases not only among young generation but also among professionals, the subject has become the thrust area of study in recent times. The subject has gained further momentum in its new incarnation under the names of 'individual processes' or 'self-management' in the institutes of management. In either situation, the methodology adopted is the one that is based on statistical analysis of data or on a vast variety of case-studies. This leads to empirical theories for the subject. Unless an individual is defined and analysed at the most fundamental level in terms of EOLs and EFLs, a formal theory for the subject remains a far cry. In its new incarnation, no doubt the subject has started dealing with very subtle and somewhat intriguing cases, but the approach remains an empirical one, mainly because the casestudies supply the basic facts only in a piece-meal manner towards the development of theories. In spite of all this, there still remains a need of a formal theory of personality. In the forthcoming sections an attempt will be made on this front using the patomic model [1] of human Being. 


\section{Revisiting the Personality Traits and Dimensions within the Frame Work of Vedic Science}

In its new incarnation, the subject has grown mostly in the West where there have been frequent industrial revolutions and the problems were cropping up between employers and employees. When studying the behavior of employees in an organization, one broadly considers five heads corresponding to five categories of processes [5] that take place, namely (a) individual processes, (b) inter-personal and group processes, (c) organizational processes, and (d) change processes, over and above them is the (e) environment, in which the employees are working. For a detailed analysis of these processes within the framework of patomic model, we shall return in Sect. 8. Here, however, we make some brief observations on the prevailing studies carried out now mostly in a scientific spirit in the institutes of management,

\section{Personality And Individual Processes}

Obviously, the personality of an individual plays an important role in the dynamics of the organization as a whole. In addition to personality, the other aspects of study under the caption of 'individual processes' pertain to one's perception, values and attitudes, learning capacity and its reinforcement or implementation, work motivation, and the last but important one is the capacity of bearing the work stress. Many of these aspects clearly rely on the fact as to how an individual has created his personality out of its subjective content, i.e., the character of the person. Before proceeding further, let us look at some modern definitions of the personality of a person.

As such there is no unique definition of personality. However, according to Engler (1991), personality of a person represents personal characteristics that lead to conscious patterns of behavour. One analyses these behavioural patterns when the person interacts with other persons. According to another definition by Hogan (1991), personality refers to relatively stable pattern of behaviours and consistent internal states that explain a person's behavioural tendencies. Further, the personality is considered to have both internal and external elements. The external traits in fact are observable behaviours that help in identifying one's personality. The internal states, on the other hand, represent the thoughts, values and genetic characteristics that one infers from the observable behaviours.

One way to understand the personality of an individual is to understand both internal and external elements that the individual has in common with others and what makes that particular individual unique. Thus, each employee in an organization is unique but may or may not respond as others do in a given situation. In fact, the theories of personality are developed mainly on this ground. Since the behavior is an outcome of stable personality traits, some social scientists do not accept the notion of personality as such instead they argue that the behavior can be determined exclusively by the external conditions. Moreover, stable traits do exist and these lead people to behave consistently with time and in different circumstances. In such descriptions, though the concept of personality appears illusive, but in reality it is not so; it remains in the background while understanding the traits of the individual. Like this, there are many other aspect of one's personality which have been the subject of study in behavioural science. 


\section{Revisiting the Personality Traits and Dimensions within the Frame Work of Vedic Science}

However, we refrain ourselves from going into these details here. Yet, some pertinent points are worth mentioning here:

(i) Hogan's definition of personality is somewhat more intriguing in the sense that it accounts for internal states and that too the consistent ones. This clearly points to the dynamics of inner EOLs in the present context.

(ii) When the behavior patterns of a person are compared with those of others, it still remains a case of relative studies, may be good for an organization but not on a absolute scale. For the absolute scale, every society or culture, however, has certain standards to judge the merit of one's personality. This standard is normally defined after keeping in mind the social and/or religious norms. For example, one can use the Raj Yoga of Patanjali as a standard [3].

(iii) The external environment in general may consist of humans, nonhumans and inanimate objects. The interactions of a person with them will, however, differ from person to person. Thus, an account of external environment, when comparing the personalities is not that simple as considered in the organizational studies.

(iv) Hofstede's framework (1980) can be presented as follows: In tune with point (ii) above, culture also provides more than an understanding of personality development. In fact, without a cultural-religious framework, the behavior of a person and hence his personality has no meaning and without meaning behavior has no purpose. It is the culture that provides people with a set of values and assumptive beliefs as well as implicit inferences about how the world operates. Thus, the culture of the society or the country a person lives in, also has an impact on the personality.

\section{Understanding personality traits and dimensions}

With a view to differentiating, ordering and naming the differences in people's behavior and experience, the concept of personality factor has been introduced in the multi-dimensional model of personality. Pertaining to human qualities, the words which were semantically similar (synonyms) were grouped in some minimal number of 16, as antonym pairs. These sixteen personality factors so found are as follows: (i) apprehensive vs. self-assured, (ii) assertive vs. humble, (iii) conscientious vs. expedient, (iv) controlled vs. casual, (v) emotionally stable vs. unstable, (vi) experimental vs. conservative, (vii) happy-go-lucky vs. sober, (viii) imaginative vs. practical, (ix) more intelligent vs. less intelligent, (x) outgoing vs. reserved, (xi) relaxed vs. tense, (xii) reserved vs. warm, (xiii) shrewd vs. forthright, (xiv) suspicious vs. trusting, (xv) tough-minded vs. sensitive, and (xvi) venturesome vs. timid. For the analysis personalities these 16 factors are further reduced to five which, in turn, have helped in developing the so-called 'Big-Five' (multidimensional) personality theory. These Big Five factors identified are: (a) extraversion (comfort level of individual), (b) agreeableness (individual's behavior towards others), (c) conscientiousness (extent to which one shows consistent and reliable behavior while working in organization), (d) emotional stability (ability to control his emotions at the time of crisis), and (e) openness to experience (ability to be open to any kind of experience that helps 
him to do the job). Each of these factors helps in understanding the behavioural patterns of one's personality.

Another approach for the personality test, based on cognitive styles of psychology and very widely used in business organizations, is due to Jung (1933). Cognitive style describes the way in which an individual perceives and processes information. According to Jung, individuals develop, mostly unconsciously, preferred ways of gathering information and evaluating it to make decisions. The two opposite ways of gathering information are through a sensing method and by intuition, and the two opposite ways of evaluating information are thinking and feeling, as depicted in Fig.1. These different ways of gathering and evaluating information combine to form matrix of four problem-solving behaviours in cognitive psychology, namely sensation thinkers (ST), intuitive thinkers (IT), sensation feelers (SF), and intuitive feelers (IF). Fig.1 demonstrates as to how these four personality types emerge. Each type has its merits and demerits, but in a complex organization all are necessary.

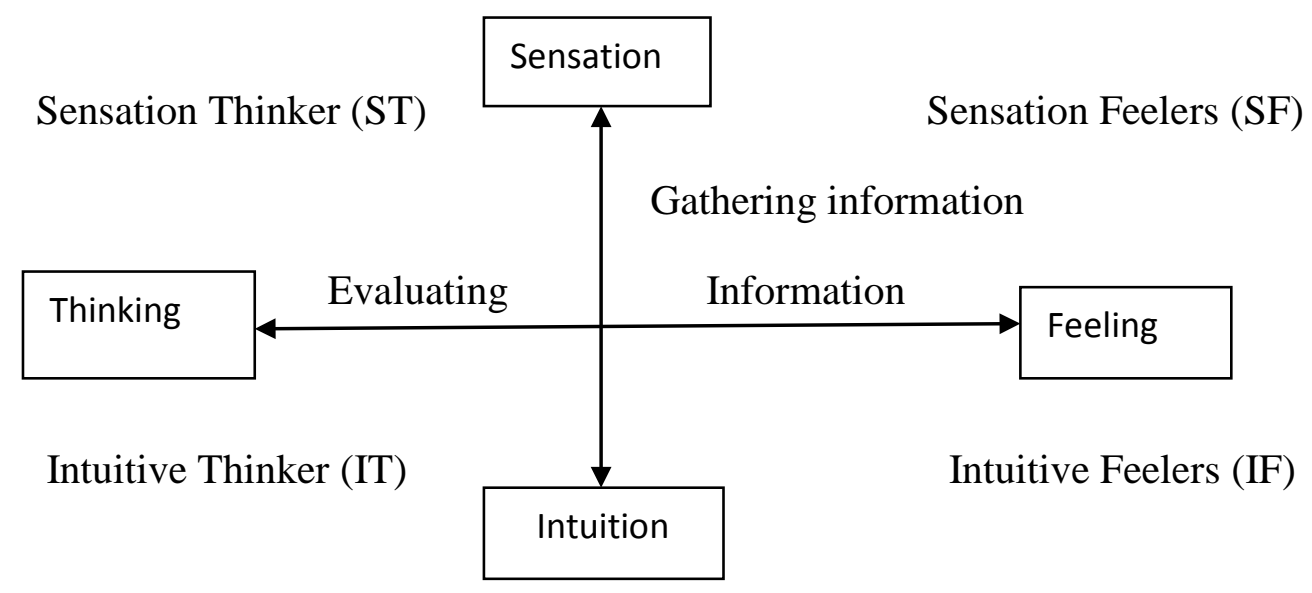

Fig. 1: The framework of Jung for handling information. In this representation, the four quadrants depict the four cognitive styles of analyzing the personality of individuals.

Further note that while using these types in the analysis of personalities of individuals, the roles of personality of managers, judges, or recruiters cannot be ruled out, and the same does not appear clearly in the theory of Jung. In the next section we make some more observations on this theory.

\section{SOME REMARKS ON THE PERSONALITY THEORY OF JUNG: A POSSIBLE MODIFICATION}

In what follows, we make some observations on the personality theory of Jung and suggest some possible modifications to it in the light of patomic model of human Being [1, 2]. 


\section{Revisiting the Personality Traits and Dimensions within the Frame Work of Vedic Science}

(i) The basic requirement to develop preferred ways of gathering information and evaluating it to make decision by a person is that he has to be conscious. A dead body can not undergo the processes of sensation, feeling, intuition and thinking. The respective agencies (faculties) responsible for these processes also have to be functional and conscious. This consciousness or awareness may vary from person to person but cannot be zero.

(ii) In fact, in Fig.1, a simple replacement of 'sensation' by the biological body and senses of knowledge $(B+S E=G)$, 'feeling' by the faculty of emotion, i.e. the mind $M$, 'intuition' by the faculty of memory, i.e. the ego E, and 'thinking' by the faculty of decision, i.e. the intellect I, as displayed in Fig.2, will help a lot in understanding and implementing the theory of Jung.

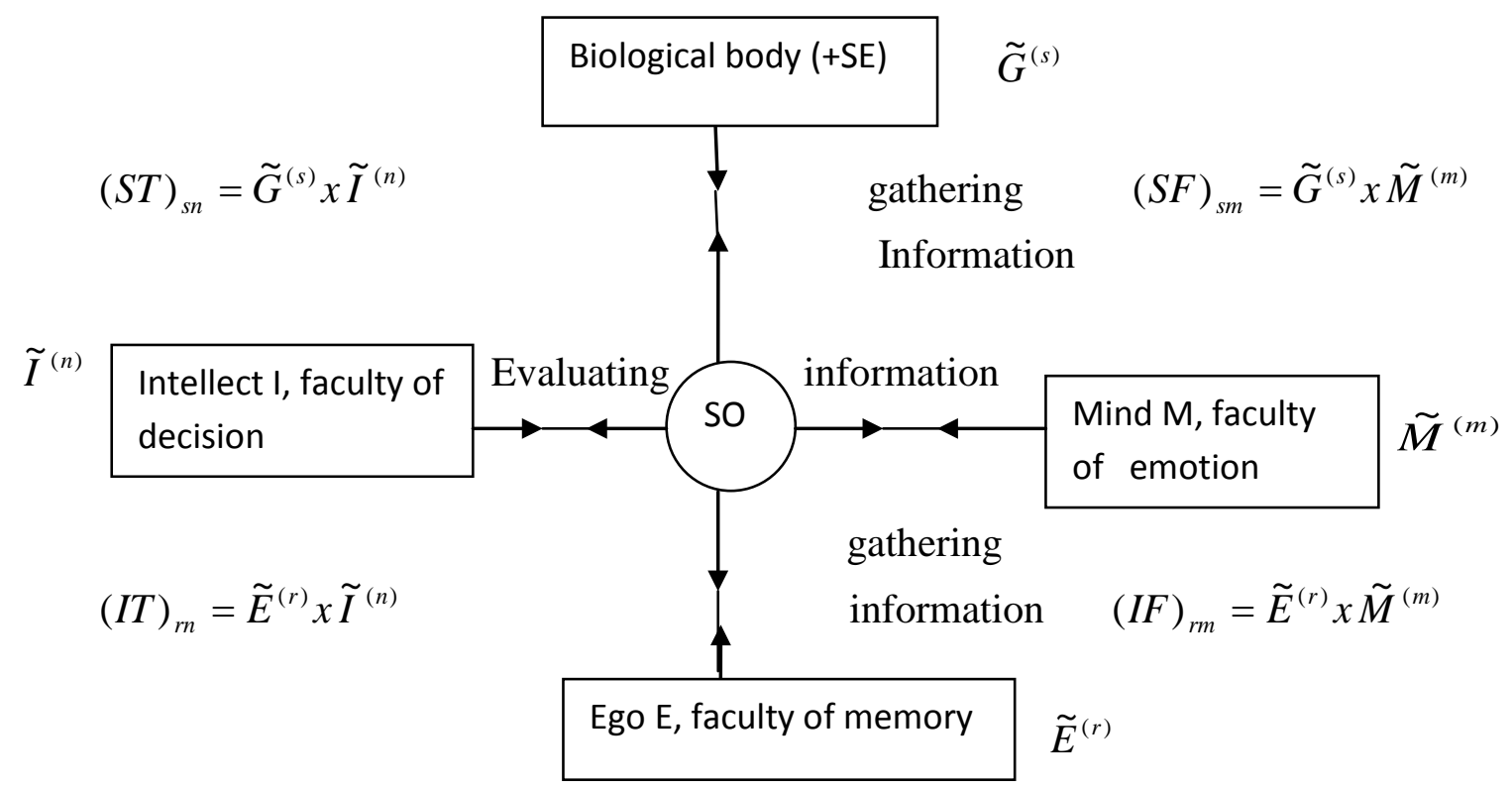

Fig. 2: Possible modifications to the personality theory of Jung in the light of patomic model of human Being. Matrix formation, which helps in distinguishing the personalities, can clearly be seen in this picture in all the four quadrants.

(iii) Since a person looking for an opportunity has to be conscious enough, it is worthwhile to consider the dressed versions each of his G. M, E and I with consciousness up to certain orders, say, respectively up to s, m, r and n orders as $\tilde{G}^{(s)}, \tilde{M}^{(m)}, \widetilde{E}^{(r)}$ and $\tilde{I}^{(n)}$ (cf. Fig.2). (See Ref.(2) and Appendix A for the concept of dressing and for the dressing rules of EOLs with consciousness).

(iv) There is no point in calling thinking and feeling as opposite ways of evaluation information, they are rather complementary to each other in view of the dressing rules (cf. Eqs.(A.1)-(A.3)). Moreover, if they are the opposite ways, then there will never be a stability- emotional or 


\section{Revisiting the Personality Traits and Dimensions within the Frame Work of Vedic Science}

otherwise (cf. Big-Five theory). Therefore, not only I and M and also G and E pairwise have to be in harmony but also each of the four has to be in tune with the source of consciousness SO.

(v) To illuminate or make functional all the four agencies constantly with consciousness the source SO plays a central role in Fig.2. This fact is shown by both-way arrows between each pair of agencies.

Such a scheme of study not only offers rather a deeper foundation to the theory of Jung but also suggests a way out to construct four-style matrices in cognitive psychology in precise mathematical terms, viz.,

$$
(S F)_{s m}=\tilde{G}^{(s)} \times \tilde{M}^{(m)} ;(I F)_{m r}=\tilde{M}^{(m)} \times \tilde{E}^{(r)} ;(I T)_{n r}=\tilde{I}^{(n)} \times \tilde{E}^{(r)},(S T)_{s n}=\tilde{G}^{(s)} \times \tilde{I}^{(n)} .
$$

where $\tilde{G}^{(s)}, \tilde{M}^{(m)}, \widetilde{E}^{(r)}$, and $\tilde{I}^{(n)}$ are the dressed versions of the corresponding agencies as discussed in Appendix A. Next we discuss the subjective aspect of personality, which, in turn, pertains to the character building of an individual.

\section{PERSONALITY AND ITS INGREDIENTS: A SCHEMATIC PICTURE}

In this section we proceed to analyse certain features of personalities within the framework of patomic model of human Being, particularly with reference to the dynamics of inner EOLs. A flowchart to this effect is shown in Fig. 3, which in some sense is the modified version of the one discussed by Parthasarathy [6]. If a person is conscious enough to undergo self-observation and watch the nature of sankalpās, then this chart works, otherwise for a common man engaged badly in worldly objects, Parthasarathy proposes [3,6] another broad classification of personalities.

In Fig. 3 the role of each EOL is clearly demonstrated in creating a personality of an individual. The process starts at the level of E through the nature (in terms of gunās) of a sankalpa and accordingly the gross body $\mathrm{G}(=\mathrm{B}+\mathrm{SE})$, mind $(\mathrm{M})$, or intellect (I) come into action, though not in isolation but in a dominating manner. For example, if $\mathrm{G}$ dominates over $\mathrm{M}$ and $\mathrm{I}$, then it leads to a personality rich in bodily qualities which manifest through his actions and perceptions but in a limited sense. Similarly, if $\mathrm{M}$ dominates over $\mathrm{G}$ and $\mathrm{I}$, then it leads to an emotional personality through feelings and other such emotions. However, the role of I is important in bringing the spiritual content in the personality. 
Revisiting the Personality Traits and Dimensions within the Frame Work of Vedic Science

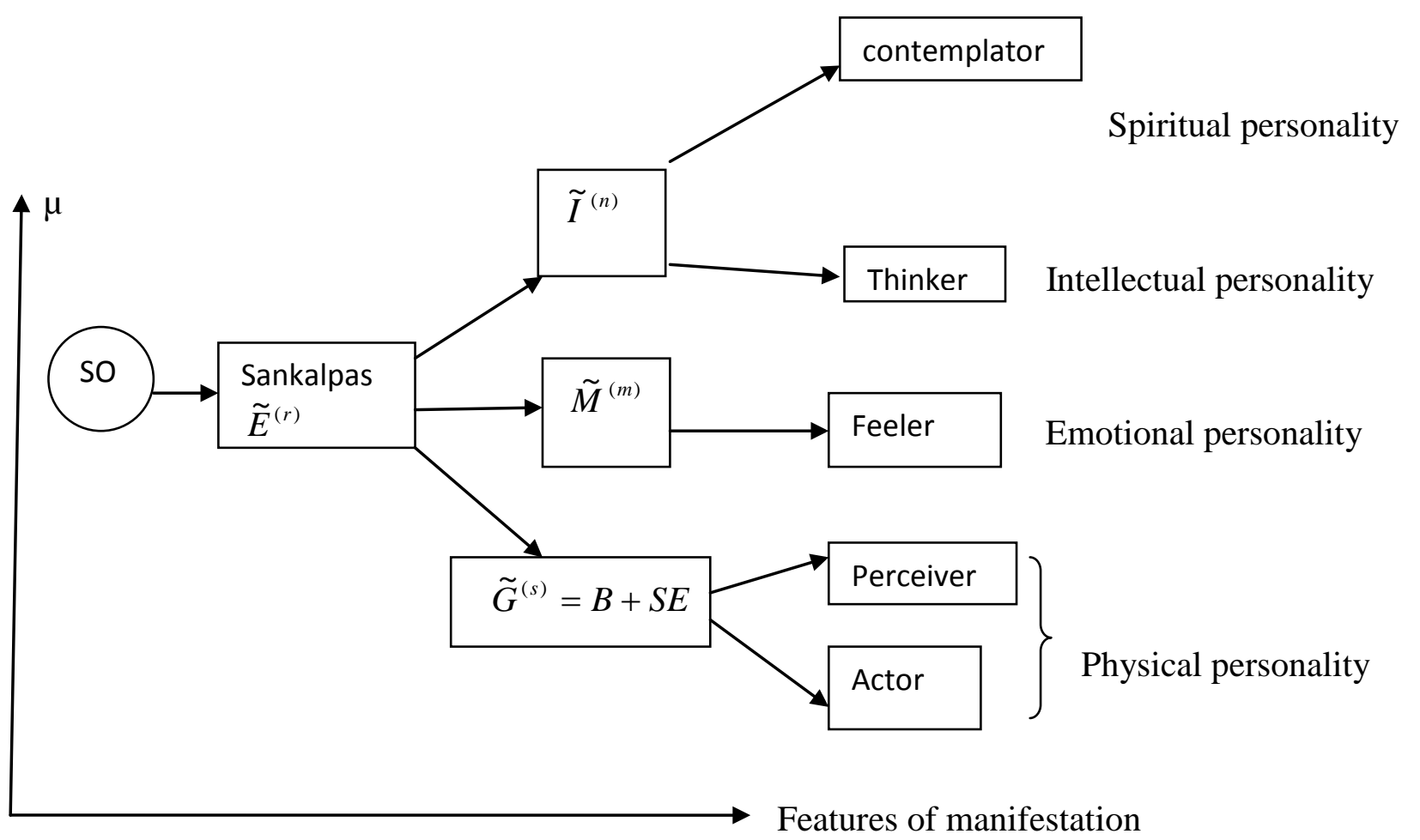

Fig. 3: A flow chart explaining the creation of different types of personalities in an individual depending on the levels of development of his FOU.

In fact, if the intellect $\mathrm{I}$ is tuned to think dominantly over $\mathrm{G}$ and $\mathrm{M}$ and to the acceptance of spatio-temporal character of the outer world, then such a thinking would lead to intellectual personality (say, that of scientist-category of persons). On the other hand, if I is tuned to think beyond the spatio-temporal character of the outer world by way of realizing (i.e. using its subtle component) the transcendental reality behind its working, then such a contemplation leads to a spiritual personality (say, that of yogi-category of persons). According to Parthasarathy [6], the subtle intellect is the discriminating faculty which contemplates upon and distinguishes the transcendental reality from the terrestrial world. Note that the nature of a sankalpa also plays role at this stage.

\section{HOW DO PERSONALITIES DIFFER?}

Whether it was the scheme of study of Jung (cf. Fig. 1) for the personality test or the original description due to Parthasarathy [6] for the creation of personality, both define four types of personalities in a broad sense. Of course they are not independent of each other. Even among these four types, there can be further sub-divisions of personalities and to account for them both schools of thought offer certain possibilities. Here, however, we have suggested a simple way to account for these differences, i.e., by replacing the nascent version of EOLs by the dressed ones. It is the order of dressing of an EOL with consciousness (cf. Appendix A) or the extent of evolution of one's EOLs with meditation that will further make a difference among the personalities of a given category. For example, there may be a variety of each of spiritual,

(C) The International Journal of Indian Psychology, ISSN 2348-5396 (e)| ISSN: 2349-3429 (p) | 64 


\section{Revisiting the Personality Traits and Dimensions within the Frame Work of Vedic Science}

intellectual, emotional, and physical category of personalities (cf. Fig. 3) and the same can be understood if one considers different orders of dressing or the development of the corresponding EOL with reference to meditation. In fact, this evolution of EOLs differs from person to person.

Another aspect emphasized in Fig. 3 which may help in differentiating the personalities is to view the personality creation scheme as a whole in the positive quadrant of $\mu$ vs. features of manifestation plane. Clearly, as the intensity of meditation (self-observation) enhances then the personality of a person may change from the physical to emotional, intellectual and then to the spiritual level. Similarly, features of manifestation in the personality increase as one proceeds from the zones of subtlest (soul), subtler (E, I and M), subtle (their functions), and finally to the gross and grossest features as shown in Fig. 3. In fact, such considerations in the creation aspect suggest the ways to improve one's own personality.

Example : Even among spiritual persons there is a spectrum of their realization, among scientist and philosopher category of persons or among the so-called skilled workers there is spectrum of their intellectual evolution, and thus each person has a varying view of the same world-order; all this creates sub-division of personalities.

It may be mentioned that the Asthanga yoga of Patanjali in terms of its components and subcomponents can also help in explaining the sub-divisions of personalities [3].

\section{IMPROVING A GIVEN PERSONALITY: CHARACTER BUILDING}

Character building means inculcating the human (both moral and ethical) values in one's way of life as defined by the norms of a society and at a given point of time. Although it is not a difficult task for an individual to do so, yet limits on such achievements are suggested by the Vedic science. As far as the nature and fruit of actions of a person are concerned, there are three basic reasons to classify them, namely prärabdha, sancit and kriyamāna which respectively means, destined out of the previous or past birth(s), performed in this birth itself and the ones performed in the present. Thus, the efforts can be made to build the character or to improve the personality and accordingly the success can be achieved to the extent that the actions confine only to the domains of sancit and kriyamanna category. Hardly any change is possible with regard to the fruit of actions of prärabdha category, as it is all destined in advance to a great extent.

To this effect, first of all there has to be a desire to improve upon the character. If it is there, then there is a huge list of human qualities to be inculcated towards this improvement of personality in all the three $\mathcal{G}, \boldsymbol{M}$ - and $\boldsymbol{C}$-domains. Some such qualities which a conscious person need to inculcate in his character are: truth, nonviolence, non-stealing, continence, abstaining from vilification, modesty, un-haughtiness, artlessness, purity, contentment, endurance, spirit of service, sacrifice, charity, austerity, study of religious scriptures, mind-control, sense-control, 


\section{Revisiting the Personality Traits and Dimensions within the Frame Work of Vedic Science}

humility, straightforwardness, compassion, faith, discrimination between true and false, dispassion, living in seclusion, non-accumulation of wealth and property, lack of doubt and distraction, minimization of desire, forgiveness, patience, absence of malice, fearlessness, pridelessness, peace, faith in a deity, etc. One can adopt some or all of them at a time and try to improve his character and subsequently the personality. Note that this spectrum of human qualities includes those of all the three $\mathcal{G}$, $\boldsymbol{M}$ - and $\boldsymbol{C}$-domains and one can pick any or all of them conveniently to go from the level of physical personality to that of the spiritual one.

Note that for these changes in personality space and time considerations are immaterial, i.e. the change can occur anywhere and at anytime as and when a person is inspired by an event or incidence of the outer world, and develops a will power for the change, or when opts for a better sankalpa, or when certain events constantly pinch him during the self-observation. Many examples can be traced from day-to-day life to this effect.

As per Vedic science the senses in the body are very powerful as far as the attachment to their respective sense-object is concerned. In fact mind joins this attachment and moulds the intellect accordingly (cf. SMBG, Verse 2.67). Conversely, if the intellect is powerful enough (i.e. dressed highly with consciousness) then the flow of energy towards the sense-objects can be controlled and the working of the (body) system becomes ordered from the so-called the chaotic one. Even this orderliness of the whole body-system can be of different categories depending upon the nature of intellect I and that of sankalpās in terms of three gunās, namely sātvik, rajas and tamas. We analyse this mechanism in the next section and argue as to how a particular case of this mechanism yields the integration of body, mind and soul.

\section{PERSONALITY CREATION MECHANISM AND THE INTEGRATION OF BODY, MIND AND SOUL}

According to Vedic science the words body, mind and soul in the phrase 'integrating body, mind and soul' respectively stand for the gross $(\mathcal{G})$, micro $(\boldsymbol{M})$ and causal $(\boldsymbol{C})$ existences of the human Being. Their integration is in the spiritual sense and the same, in fact, is an idealized situation which is not easy to achieve by an individual everywhere and at all times. No doubt, there is always a coordination of body, mind and soul towards fruit or success of actions but their integration is a particularized case of this coordination in the sense that what one thinks, so he expresses in words and acts in practice accordingly. In fact, the word integration is used in two related senses, namely (i) in the sense of strengthening the body, mind and soul towards the success of an action, and (ii) to have straightness in all activities of mind, senses and the body. Any way both these purposes are useful as far as the development of personality is concerned. Any departure from this policy does not justify the integration of body mind and soul. It is only in this situation that one can make best use of the energies associated with his body, mind and soul. This indeed creates an ideal condition to ensure success in the actions so performed. 


\section{Revisiting the Personality Traits and Dimensions within the Frame Work of Vedic Science}

Now question arises as to what type of actions need to be performed with reference to the maintenance of world-order and the value system in a society? Naturally, one does not want to spoil the world or the Universe as a whole- a beautiful gift of Mother Nature. Thus, the above idealized situation should be the goal of one's life and the integration of body, mind and soul should be taken in the spirit of positive pursuits [2,3] and spiritual sense. In what follows, we discuss a personality creation mechanism (PCM) in the light of Vedic science and argue that the integration aspects of body, mind and soul are just a particular case of this general theory.

Although one can start with the dressed versions of inner EOLs and analyse the case of the finest possible processes taking place inside the human Being, but here we restrict ourselves only to the nascent versions of EOLs (cf. Appendix A). However, as we consider the role of three gunās in the mechanism, this in some sense will bring in certain orders of dressing of EOLs with consciousness. Here we discuss a Jung-type (cf. Sect. 3) matrix formation at the level of character building, which at a later stage affects the personality so developed.

Note that either due to prārabdha or due to certain in-born qualities of an individual a sankalpa (S) can come in three colors or is influenced by the three gunās, namely sātvik (s), rajas (r), tamas (t) to furnish three possibilities $\mathrm{S}_{\mathrm{s}}, \mathrm{S}_{\mathrm{r}}, \mathrm{S}_{\mathrm{t}}$. On the other hand, the guiding agency (intellect) I, which for an ordered working of the body-system plays an important role in the actions performed by the body $\mathrm{G}$ through the mind $\mathrm{M}$, can also comes in three abstract colors as $\mathrm{I}_{\mathrm{s}}, \mathrm{I}_{\mathrm{r}}, \mathrm{I}_{\mathrm{t}}$. Now, in order to account for all the possibilities, one can construct the so-called personality matrix $\mathrm{P}$ from the sankalpa matrix $\mathrm{S}$ and the intellect matrix I, viz. $\mathbf{P}=\mathbf{S} \times \mathbf{I}$, as

$$
P=\left(\begin{array}{lll}
S_{s}-I_{s} & S_{s}-I_{r} & S_{s}-I_{t} \\
S_{r}-I_{s} & S_{r}-I_{r} & S_{r}-I_{t} \\
S_{t}-I_{s} & S_{t}-I_{r} & S_{t}-I_{t}
\end{array}\right) \equiv\left(\begin{array}{lll}
(S I)_{s s} & (S I)_{s r} & (S I)_{s t} \\
(S I)_{r s} & (S I)_{r r} & (S I)_{r t} \\
(S I)_{t s} & (S I)_{t r} & (S I)_{t t}
\end{array}\right)
$$

Following remarks are in order about the nature of this construction:

(i) Note that $\mathrm{s}, \mathrm{r}, \mathrm{t}$ are the independent variables and constitute a space (may be Cartesian in mathematical sense) in which both sankalpa and intellect are projected.

(ii) There are three zones of integration of body, mind and soul: The nine elements of the matrix $\mathrm{P}=\mathrm{SI}$ represent nine types of characters or personalities, out of which the diagonal ones, namely $(\mathrm{SI})_{\mathrm{ss}},(\mathrm{SI})_{\mathrm{rr}}$ and $(\mathrm{SI})_{\mathrm{tt}}$ correspond to the integrated body, mind and soul of an individual. The integration can be there for all the three category of persons, namely, (i) for a sātvik person, this in turn will take him to the spiritual zone, (ii) for a rājasik person, this in turn will make him a successful person in worldly matters by maintaining no harm to Nature, and (iii) for a tāmasik person, this in turn will take him to a downfall with reference to the world-order and society.

(iii) In general it is the sankalpa that influences the intellect and not the vice versa. Therefore, $\mathrm{SI} \neq \mathrm{IS}$, i.e. the matrices $\mathrm{S}$ and I do not commute. However, for a man of 
perfection or for a yogi of highest degree both $\mathrm{S}$ and I stand in the same zone of consciousness [2] and they can commute, i.e. SI=IS, only for this exceptional case.

(iv) Nonintegrated personalities: There are six categories of nonintegrated personalities corresponding to six off-diagonal elements in the personality matrix P, which are (1) persons improving from rājasik to sātvik zone correspond to $(\mathrm{SI})_{\mathrm{sr}}$; (2) persons improving from tāmasik to sātvik zone- a bit difficult task, correspond to (SI) $)_{\mathrm{s}}$; (3) persons improving from tāmasik to rājasik zone correspond to (SI) $)_{\mathrm{rt}}$; (4) persons degrading themselves from sātvik to rājasik, correspond to (SI)rs; (5) persons degrading themselves from sātvik to tāmasik, correspond to (SI) $)_{\mathrm{ts}}$, and (6) persons degrading themselves from rājasik to tämasik, correspond to $(\mathrm{SI})_{\mathrm{tr}}$. These categories are further listed in Table 1 (cf. category (b)).

Table 1: Classification of personalities on the basis of Vedic science.

\begin{tabular}{|c|c|c|}
\hline S.No. & Category of personality & Nature of personality \\
\hline (a) & Integrated personalities & $\begin{array}{l}\text { (1)Satoguni sātvik persons } \\
\text { (2)Rajoguni rājasik persons } \\
\text { (3)Tamoguni tāmasik persons }\end{array}$ \\
\hline (b) & Non-integrated personalities & $\begin{array}{l}\text { (4)Rajoguni sātvik persons } \\
\text { (5)Tamoguni sātvik person } \\
\text { (6)Tamoguni rājasik persons } \\
\text { (7)Satoguni rājasik persons } \\
\text { (8) Satoguni tāmasik persons } \\
\text { (9) Rajoguni tāmasik persons }\end{array}$ \\
\hline (c) & Ideally integrated personalities & $\begin{array}{l}\text { (10) Satoguni sātvik persons with } \\
\text { zero nonintegrated component } \\
\text { (11) Rajoguni rājasik persons with } \\
\text { zero nonintegrated component } \\
\text { (12) Tamoguni tāmasik persons } \\
\text { with zero nonintegrated } \\
\text { component }\end{array}$ \\
\hline
\end{tabular}

(v) Integrated personalities of Remark (ii), in whichever zone they are, have stable mind at least for a certain period of time and at a given place. On the other hand, the nonintegrated personalities of Remark (iv) are of fluctuating mind, always undergoing transitions from one type nature to another type.

(vi) In view of Remarks (iv) and (v), the personality matrix $\mathrm{P}$ is not symmetric i.e., $(\mathrm{SI})_{\mathrm{ij}} \neq(\mathrm{SI})_{\mathrm{ji}}$, where $\mathrm{i}, \mathrm{j}=\mathrm{s}, \mathrm{r}, \mathrm{t}$ and $\mathrm{i} \neq \mathrm{j}$. As a matter of fact when a person undergoing transitions from one zone of gunās to another zone, the circumstances and environment of the person change. Therefore, a change in personality is bound to exist in spite of the forward and backward transition of the same nature. 


\section{Revisiting the Personality Traits and Dimensions within the Frame Work of Vedic Science}

(vii) A better lot of integrated personalities: Integrated personalities of remark (ii) correspond to the fact that the nature of person in terms of gunās whether sātvik, rājasik or tämasik is sharply defined; otherwise most of us are born with mixed gunas, but dominated by any one or two of them. Therefore, it is worthwhile to look for the diagonalized version of the matrix $\mathrm{P}$ as $P_{D}$, viz.,

$$
P_{D}=\left(\begin{array}{ccc}
(\overline{S I})_{s s} & 0 & 0 \\
0 & (\overline{S I})_{r r} & 0 \\
0 & 0 & (\overline{S I})_{t t}
\end{array}\right),
$$

where the integrated personalities corresponding to $(\overline{S I})_{s s},(\overline{S I})_{r r}$ and $(\overline{S I})_{t t}$ are truly the integrated ones in practical terms. In fact one can attempt to find the diagonalizing matrix whose elements will be the appropriate mixtures of different gunās. Further, this mixing can be attributed to the effect of circumstances and environment in which the individual is placed. We can place this class of personalities in ideally integrated personalities as there is no energy loss in any other dimension of human activities. For the role of trinity of gunās in personality studies, we refer to some recent work of Singh, Mishra and Raad [7] and also of Parthasarathy [8]. Next we analyse the personalities listed in Table 1.

\section{HUMAN PERSONALITIES WITHIN THE FRAMEWORK OF VEDIC SCIENCE}

Note that the nomenclature of a personality in Table 1 refers to both the innermost, i.e. at the level of sankalpa and the exterior, i.e., at the level of intellect aspects of a personality. For example, a rajoguni sātvik personality (cf. case b4)) means the sankalpa and intellect of a person are enveloped respectively in rājasik and sātvik gunās. Further, the trinity of gunās, namely sātvik, rajas and tamas, in some sense can be considered in the spirit of superlative, comparative and positive degrees of an adjective, respectively. It is well accepted that the inner EOLs or the human characteristics enveloped in sātvik guna are the best from the point of view of both maintenance of world-order and ethical as well as moral values in a society. In fact, the inner EOLs enveloped in sātvik guna lead to actions in the outer world which are the best in the maintenance of world-order. Contrarily, the roles of EOLs or of human characteristics enveloped in tāmasik guna in human behavior and actions are not well appreciated in a society and also not considered to be good in the maintenance of world-order. In a nut-shell, it is not wrong to say that the positivity at any level leads to order in the Universe whereas the negativity leads to chaos in the Universe. 


\section{Revisiting the Personality Traits and Dimensions within the Frame Work of Vedic Science}

The three categories of personalities of Table 1, namely integrated, nonintegrated and ideally integrated can be analysed further in terms of their stability with respect to space and time. In the first category, the satoguni sātvik personality is supposed to be the best where as the tamoguni tämasik personality is considered as the worst with reference to values in a society or with regard to the world-order. In these cases there is an occasional stability in the nature of such persons and the equilibrium among the trinity of gunās keeps on changing with circumstances and environment. Nonintegrated components also manifest occasionally in these personalities, but for the most of the time there is definitely some correlation between their thinking and doing.

The second category concerns the nonintegrated personalities which are 6 in number. Among them the tamoguni rājasik and rajoguni tāmasik personalities (cf. (b6) and (b9)) are considered to be on the worse side whereas the räjoguni sātvik and satoguni rājasik personalities (cf. (b4) and (b7)) are on the better side in a relative sense. This category of persons never notices a stability of mind and thus the question of equilibrium among the trinity of gunas does not arise. Often there appears a kind of nonlinearity between their thinking and doing. These personalities keep on changing their decisions with environment and circumstances.

Clearly, the third category of ideally integrated personalities is the most stable ones with respect to space and time. Also, they are not affected easily by the changed environment and circumstances. This is mainly because a kind of equilibrium among the trinity of gunās is already established in an ideal and appropriate manner prior to the final achievement. Even in this category, satoguni sātvik personality is the best and the tamoguni tāmasik is the worst with regard to the standards set in a society in spite of the fact that there is a linearity and simplicity between the thinking and the acting of persons of all the three categories.

Further it is noticed that the personality of a person is not stable during his learning stage, i.e., when he is a student or becomes a youth. However, only after attaining the adulthood, his personality tends towards the stability and the changes are slow but steady, if at all they are there. Many examples of these categories can be found in the society. However, we refrain ourselves from such details here.

\section{CONCLUDING DISCUSSION}

With a view to giving a better foundation to the subject of personality and its development in a person, several suggestions are advanced in this paper within the framework of the patomic model. To this effect, commonly used theory of Jung for the personality test takes a new look when the agencies of gathering information and evaluating information in the work of Jung are pairwise replaced by gross body $(\mathcal{G})$ and ego $(\mathrm{E})$ and by mind $(\mathrm{M})$ and intellect (I), respectively. Latter are the basic ingredients of the patomic model which contribute to the dynamics that takes place inside the human Being not only before but also during his performance of an action. Further replacements of these EOLs by their dressed versions with consciousness (cf. Sect. 3) clearly explain, in a very general manner, the matrix formation corresponding to the four

(C) The International Journal of Indian Psychology, ISSN 2348-5396 (e)| ISSN: 2349-3429 (p) | 70 


\section{Revisiting the Personality Traits and Dimensions within the Frame Work of Vedic Science}

cognitive styles of psychology namely sensation feelers, intuitive feelers, sensation thinkers and intuitive thinkers.

Alternatively, within the frame work of a similar scheme due to Parthasarathy which is also based on the concepts of Vedic science, the ingredients of a personality are identified and their role in the dynamics of EOLs is analysed (cf. Sect. 4). Viability of this scheme is also demonstrated in classifying the personalities and their tests. It is further argued that the personalities of persons differ on the basis of different orders of dressing of their different inner EOLs with consciousness. Using again the concepts of Vedic science, some ways to improve the character and subsequently the personality of a person are highlighted (cf. Sect. 6). In particular, the role of gunās of Vedic science in the functioning of inner EOLs is explored. This forms the basis for the newly proposed personality creation mechanism. In mathematical terms, a personality matrix is formed to this effect, whose nine components accordingly define nine types of personalities in general (cf. Table 1). Out of these nine, three types are the integrated ones and the rest six are the non-integrated. One can as well classify the personalities on this basis and prepare a questionnaire to this effect. Compared to the scheme of Jung, this kind of personality test will definitely reflect a long-term stability in the nature of a person. As far as the integration of body, mind and soul towards the performance of an action is concerned, it appears as a particular case of this general analysis. It is emphasized that a mixed nature of gunās in the personality will give rise to a better integrated personality as far as the stability of mind is concerned. Further, note that such an integration of EOLs is suggested to exist in different psychic zones corresponding to sātvik, rājasik and tāmasik nature of a person. The subject matter in fact requires further analysis and case studies. We restrict ourselves from going into these details here. However, it may be mentioned that on the personality of a person there is also a great impact of culture and environment in which he lives in. An account of these factors should also be taken care of in such studies.

\section{Acknowledgements}

The Post-retirement facilities from Ramjas College, University of Delhi, are gratefully acknowledged. Thanks are also due to Dr. D. Parashar (physics) and Prof. Girishwer Mishra (Psychology) for many useful discussions.

\section{NOTES AND REFERENCES}

1. Radhey Shyam Kaushal, "The Philosophy of the Vedanta: A Modern Scientific Perspective” (Garib Dass Oriental Series No. 179, Indian Book Centre, Delhi, 1994). Also see, "Patomic Model of Human Being in the Context of Modern Science", Ph.D. Thesis (unpublished), University of Delhi, 2000 ; "Human Being: From the Point of View of a Philosophical Atom-type Model”, Journal of Scientific \& Industrial Research (New Delhi) 49 (1990) 578-82. 


\section{Revisiting the Personality Traits and Dimensions within the Frame Work of Vedic Science}

2. Radhey Shyam Kaushal, "The Science of Philosophy: Theory of Fundamental Processes in Human Behaviour and Experiences”, ( D. K. Print World, New Delhi, 2011)

3. Radhey Shyam Kaushal, "Psychophysics and Human Interactions: Art and Science of Integrating Body, Mind and Soul”, (New Age Books (Motilal Banarasidas), New Delhi, 2015); The present paper is an abridged version of Chapter 9 of this book.

4. Srimadbhagvad-Gita, See any Edition, Gita Press, Gorakhpur (Abbreviated as SMBG in the Text).

5. See, for example, Kavita Singh, "Organizational Behaviour: Text and Cases” (Pearson Education, Dorling Kindersley (India), Pvt. Ltd., Delhi, 2010)

6. A. Parthasarathy, "Vedanta Treatise”, (Vedanta Life Institute, Bombay, 4th ed., 1992).

7. Jitendra K. Singh, Girishwar Mishra and Boele De Raad, "Personality Structure in the Trait Laxicon of Hindi: Major Language Spoken in India”, Preprint, November 2012, ( to appear in European Journal of Personality).

8. A. Parthasarathy, “Governing Business and Relationships”, ( Vedanta Life Institute, Bombay, 2013).

\section{APPENDIX A: DRESSING RULES OF INNER EOLS WITH CONSCIOUSNESS}

As per common experience the EOLs of a person work when they are illuminated by or enlightened with the consciousness, i.e. in the presence of soul. Not only this, an external object is perceived when only conscious EOLs participate in the process of perception [2]. This in fact is the basis for the pă̌cikaran rule for creating perceived mahābh ütās from the physical (nascent) ones in Vedic science. Now the question is as to how the EOLs, particularly the inner ones, $\mathrm{M}$, I and E get dressed with the consciousness emanating from the soul. To this effect, the author has proposed earlier [2] a 'trikaran rule (in analogy with the pa ̌̌ci karan rule for PMs) which takes care of dressings of inner EOLs M, I and E.

Note that the increasing consciousness means more and more intense meditation, characterized by the variable $\mu$. Further, note that the dressing of an EOL or of an external object with consciousness tantamount as to how these entities evolve with the variation of meditation variable $\mu$. In mathematical terms, there are two ways to study this aspect, namely as discrete evolution and as continuous evolution with respect to $\mu$. While the author has studied both these ways earlier, here, however we restrict ourselves only to discrete evolution and that too of inner EOLs through a 'trikaran' rule. According to this rule, a dressed EOL is the sum of $50 \%$ of its undressed version and $25 \%$ of undressed version of each of the other two EOLs. Thus, for example, the first-order dressed versions, $\widetilde{M}^{(1)}, \quad \tilde{I}^{(1)}$ and $\widetilde{E}^{(1)}$ respectively of M, I and E can be expressed in terms of the undressed (nascent) ones by simple algebraic relations of the form: 
Revisiting the Personality Traits and Dimensions within the Frame Work of Vedic Science

$$
\begin{aligned}
& \widetilde{M}^{(1)}=\frac{1}{2} \mathrm{M}+\frac{1}{4} \mathrm{I}+\frac{1}{4} \mathrm{E}, \\
& \widetilde{I}^{(1)}=\frac{1}{2} \mathrm{I}+\frac{1}{4} \mathrm{E}+\frac{1}{4} \mathrm{M}, \\
& \widetilde{E}^{(1)}=\frac{1}{2} \mathrm{E}+\frac{1}{4} \mathrm{M}+\frac{1}{4} \mathrm{I} .
\end{aligned}
$$

Similarly, for the second-order dressing one writes

$$
\begin{aligned}
& \widetilde{M}^{(2)}=\frac{1}{2} \widetilde{M}^{(1)}+\frac{1}{4} \widetilde{I}^{(1)}+\frac{1}{4} \widetilde{E}^{(1)}, \\
& \widetilde{I}^{(2)}=\frac{1}{2} \widetilde{I}^{(1)}+\frac{1}{4} \widetilde{E}^{(1)}+\frac{1}{4} \widetilde{M}^{(1)}, \\
& \widetilde{E}^{(2)}=\frac{1}{2} \widetilde{E}^{(1)}+\frac{1}{4} \widetilde{M}^{(1)}+\frac{1}{4} \widetilde{I}^{(1)},
\end{aligned}
$$

and in general for the $\mathrm{n}^{\text {th }}$ order dressing one writes in terms of $(\mathrm{n}-1)^{\text {th }}$ order dressed EOLs as

$$
\begin{aligned}
& \widetilde{M}^{(\mathrm{n})}=\frac{1}{2} \widetilde{M}^{(\mathrm{n}-1)}+\frac{1}{4} \widetilde{I}^{(\mathrm{n}-1)}+\frac{1}{4} \widetilde{E}^{(\mathrm{n}-1)}, \\
& \widetilde{I}^{(\mathrm{n})}=\frac{1}{2} \widetilde{I}^{(\mathrm{n}-1)}+\frac{1}{4} \widetilde{E}^{(\mathrm{n}-1)}+\frac{1}{4} \widetilde{M}^{(\mathrm{n}-1)}, \\
& \widetilde{E}^{(\mathrm{n})}=\frac{1}{2} \widetilde{E}^{(\mathrm{n}-1)}+\frac{1}{4} \widetilde{M}^{(\mathrm{n}-1)}+\frac{1}{4} \widetilde{I}^{(\mathrm{n}-1)} .
\end{aligned}
$$

It is not difficult to check that at every order of dressing, one has

$$
\begin{aligned}
\widetilde{M}^{(\mathrm{n})}+\widetilde{I}^{(\mathrm{n})}+\widetilde{E}^{(\mathrm{n})} & =\widetilde{M}^{(\mathrm{n}-1)}+\widetilde{I}^{(\mathrm{n}-1)}+\widetilde{E}^{(\mathrm{n}-1)} \\
& =\mathrm{M}+\mathrm{I}+\mathrm{E},
\end{aligned}
$$

which implies that the entire process of dressing of EOLs goes in a self consistent manner, i.e. without affecting their nascent versions.

Several deductions from these simple relations have been analyzed by the author earlier (see, Ref. (2)). Interestingly, the conclusions drawn thereof, conform to many Vedic concepts. For example, one can now immediately consider a $\mathrm{n}^{\text {th }}$ order dressed micro-body as $\widetilde{M}^{(\mathrm{n})}=\left\{\widetilde{M}^{(\mathrm{n})}\right.$, $\widetilde{I}^{(\mathrm{n})}, \widetilde{E}^{(\mathrm{n})}$ \}. For the infinitely dressed case, i. e. for $\mathrm{n}=\infty$, one obtains the infinitely dressed versions of $\mathrm{M}$, I and $\mathrm{E}$ as $\widetilde{M}^{(\infty)}, \widetilde{I}^{(\infty)}, \widetilde{E}^{(\infty)}$. These later versions correspond to the cosmic mind, cosmic intelligence and cosmic desire as discussed in Ref. (3). Thus, as and when the meditation becomes more and more intense then, either one, two or all the three EOLs M, I and E tend to achieve the Supreme (cosmic consciousness) in accordance with the yoga of devotion, yoga of action and yoga of knowledge of Vedic science. For further details see Ref. (2).

How to cite this article: R Kaushal (2016), Revisiting the Personality Traits and Dimensions within the Frame Work of Vedic Science, International Journal of Indian Psychology, Volume 3, Issue 4, No. 56, ISSN 2348-5396 (e) | ISSN: 2349-3429 (p), DIP: 18.01.007/20160304, ISBN: 978-1-365-23992-2 